\title{
D-REPRESENTATION OF SUBDIFFERENTIALS OF DIRECTIONALLY LIPSCHITZ FUNCTIONS
}

\author{
ALEJANDRO JOFRE AND LIONEL THIBAULT
}

(Communicated by William J. Davis)

\begin{abstract}
Subdifferentials of convex functions and some regular functions $f$ are expressed in terms of limiting gradients at points in a given dense subset of $\operatorname{dom} \nabla f$.
\end{abstract}

\section{INTRODUCTION}

Using the theorem about the representation of a convex set in terms of its extreme points and extreme directions and considering some specific finite dimensional arguments, R. T. Rockafellar has proved in [10] the following important result: if $f: \mathbb{R}^{p} \rightarrow \mathbb{R} \cup\{+\infty\}$ is an 1.s.c. convex function with int $\operatorname{dom} f \neq \varnothing$, then

$$
\begin{array}{r}
\text { (0) } \partial f(x)=\operatorname{clco}\left\{\lim _{i} \nabla f\left(x_{i}\right): x_{i} \in \operatorname{dom} \nabla f, x_{i} \rightarrow x\right\}+N(\operatorname{dom} f ; x) \\
\forall x \in \operatorname{dom} f,
\end{array}
$$

where $\partial f(x)$ and $N(., x)$ denote the convex subdifferential and normal cone respectively. Note that in the original definition of generalized gradients for Lipschitz functions F. H. Clarke [3] considered the limiting gradient set as in the first term of the second member of $(0)$.

Generalizations of formula (0) with some limiting proximal or Fréchet subgradients in place of limiting gradients are proved by R. T. Rockafellar [13] for $\mathbb{R}^{p}$ and J. M. Borwein and H. M. Strojwas [1] and J. S. Treiman [15] for Banach spaces.

Our aim is to extend formula ( 0 ) as stated above to convex functions defined on infinite dimensional spaces and to establish a similar formula for Clarke subdifferentials of some regular functions. So we shall extend the $D$-representation result of R. Correa and A. Jofre [5].

Received by the editors May 18, 1989.

1980 Mathematics Subject Classification (1985 Revision). Primary 49A52, 46A55, 56C20.

Key words and phrases. Directionally Lipschitz functions, pseudoregular functions, weak Asplund spaces. 
Our techniques are different from those of R. T. Rockafellar [10] (for convex functions) and provide a simple proof of relation (0).

\section{Preliminaries}

Throughout this paper $E$ will be a real locally convex (Hausdorff) vector space. For $f: E \rightarrow \mathbb{R} \cup\{+\infty\}$ and $f(a)<\infty$, we shall consider the following directional derivatives (see [11], [12]):

(a) the Dini directional derivatives

$$
\begin{aligned}
& d^{-} f(a ; h)=\liminf _{t \downarrow 0} t^{-1}[f(a+t h)-f(a)], \\
& d^{+} f(a ; h)=\underset{t \downarrow 0}{\limsup t^{-1}[f(a+t h)-f(a)],}
\end{aligned}
$$

(b) the lower Hadamard directional derivative

$$
\underline{d} f(a ; h)=\liminf _{\left.\left(t, h^{\prime}\right) \rightarrow 0_{+}, h\right)} t^{-1}\left[f\left(a+t h^{\prime}\right)-f(a)\right],
$$

(c) the Clarke directional derivative

$$
f^{0}(a ; h)=\limsup _{t \downarrow 0, x \rightarrow f^{a}} t^{-1}[f(x+t h)-f(x)],
$$

where $x \rightarrow{ }_{f} a$ means $x \rightarrow a$ and $f(x) \rightarrow f(a)$, and

(d) the Rockafellar directional derivative

$$
f^{\dagger}(a ; h)=\sup _{H \in \mathscr{N}(h)} \limsup _{t \downarrow 0 x \rightarrow f^{a}} \inf _{h^{\prime} \in H} t^{-1}\left[f\left(x+t h^{\prime}\right)-f(x)\right],
$$

where $N(h)$ denotes the filter of neighborhoods of $h$.

Recall [11] that $f^{\dagger}(a ;$.$) is a lower semicontinuous (1.s.c.) convex function$ and that a considerable simplification in the analysis of $f^{\dagger}(a ;$.$) is possible in$ the case of a directionally Lipschitz function.

The function $f$ is said to be directionally Lipschitz at $a$ with respect to a vector $h([11])$ if

$$
\limsup _{\substack{\left(t, h^{\prime}\right) \rightarrow\left(0_{+}, h\right) \\ x \rightarrow f^{a}}} t^{-1}\left[f\left(x+t h^{\prime}\right)-f(x)\right]<+\infty .
$$

If this relation holds for some $h$, one says that $f$ is directionally Lipschitz at $a$.

We shall also consider the Hadamard subdifferential

$$
\underline{\partial} f(z)=\left\{x^{*} \in E^{*}:\left\langle x^{*}, h\right\rangle \leq \underline{d} f(a ; h), \forall h \in E\right\}
$$

and the Clarke subdifferential

$$
\partial f(a)=\left\{x^{*} \in E^{*}:\langle x, h\rangle \leq f^{\dagger}(a ; h), \forall h \in E\right\} .
$$

1.1. Proposition [11]. Let $f$ be directionally Lipschitz at $a$. Then

(i) $f^{\dagger}(a ; h)=\liminf _{h^{\prime} \rightarrow h} f^{0}\left(a ; h^{\prime}\right)$ for all $h \in E$

(ii) $\operatorname{int} \operatorname{dom} f^{\dagger}(a ;$.$) is the set of all vectors h$ with respect to which $f$ is directionally Lipschitz. 


\section{D-Limiting HADAMARD SUBDIFFERENTIALS}

Throughout this section we assume that $f: E \rightarrow \mathbb{R} \cup\{+\infty\}$ is lower semicontinuous (1.s.c.), $a \in \operatorname{dom} f$ and $D \subset \operatorname{dom} f$ is graphically dense in $\operatorname{dom} f$; that is, for any $x \in \operatorname{dom} f$ there exists a net $\left(x_{i}\right)_{i \in I}$ in $D$ with $x_{i} \rightarrow{ }_{f} x$. We also assume $\underline{\partial} f(x) \neq \varnothing$ for all $x \in D$, and we put

$$
\limsup _{x \in D \rightarrow f^{a}} \underline{\partial} f(x):=\left\{w^{*}-\lim _{i} x_{i}^{*}: x_{i}^{*} \in \underline{\partial f}\left(x_{i}\right), x_{i} \in D \rightarrow{ }_{f} a\right\} .
$$

2.1. Lemma. If $f$ is directionally Lipschitz at $a$ and if $r$ denotes the support function of the set cl colim $\sup _{x \in D \rightarrow f} \partial f(x)$, then

$$
r(0)=0 \quad \text { and } \quad r(h)=\limsup _{x \in D \rightarrow f^{a}} s(x ; h) \quad \forall h \in \operatorname{int} \operatorname{dom} f^{\dagger}(a ; .),
$$

where $(s(x ;$.$) denotes the greatest l.s.c convex function majorized by \underline{d} f(x ;$.$) .$

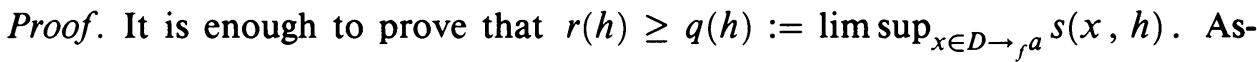
sume that $q(h) \neq-\infty$ and fix $\varepsilon>0$.

As $f$ is Lipschitz in the direction $h$, there exist $\beta \in \mathbb{R}, \delta>0$, and $V \in$ $\mathscr{N}(0)$ such that

(2) $\left.\left.t^{-1}\left[f\left(x+t h^{\prime}\right)-f(x)\right] \leq \beta \quad \forall t \in\right] 0, \delta\right], \quad \forall h^{\prime} \in h+V, \quad \forall x \in a+V$, with $|f(x)-f(a)| \leq \delta$. Choose $\left(x_{i}\right)_{i}$ in $a+V$ with $\left|f\left(x_{i}\right)-f(a)\right| \leq \delta$ and $q(h)=\lim _{i} s\left(x_{i} ; h\right)$.

By (2) we may suppose $\left|s\left(x_{i} ; h\right)\right|<\infty$ for all $i$. Moreover there exist $\gamma \in \mathbb{R}$ and $x_{i}^{*} \in \underline{\partial} f\left(x_{i}\right)$ such that

$$
\gamma \leq s\left(x_{i} ; h\right)-\varepsilon<\left\langle x_{i}^{*}, h\right\rangle \text { for all } i \geq i_{0} .
$$

As $\left\langle x_{i}^{*}, h+v\right\rangle \leq \beta$ for all $v \in V$ (because of (2)) we obtain for all $i \geq i_{0}$

$$
\left\langle x_{i}^{*}, v\right\rangle \leq \beta-\left\langle x_{i}^{*}, h\right\rangle \leq \beta-\gamma \quad \forall v \in V .
$$

So we may suppose $\left(x_{i}\right)$ is bounded and hence we may also suppose that $\left(x_{i}^{*}\right)$ $w^{*}$ converges to some $x^{*} \in E^{*}$. By what precedes we have $q(h)-\varepsilon \leq\left\langle x^{*}, h\right\rangle$ and $x^{*} \in \limsup _{x \in D \rightarrow f} a f(x)$.

Therefore $q(h)-\varepsilon \leq r(h)$ and $r(0)=0$ since $\lim _{\sup _{x \in D \rightarrow f_{f}} a} \underline{\partial} f(x) \neq \varnothing$.

2.2. Proposition. If $f$ is directionally Lipschitz at $a$, then

$$
\partial f(a)=\operatorname{clco}\left(\limsup _{x \in D \rightarrow f^{a}} \underline{\partial} f(x)\right)+N\left(\operatorname{dom} f^{\dagger}(a ; .) ; 0\right)
$$

iff

$$
f^{\dagger}(a ; h) \leq \limsup _{x \in D \rightarrow f^{a}} s(x ; h) \quad \forall h \in \operatorname{int} \operatorname{dom} f^{\dagger}(a ; .) .
$$

Proof. Observe that the right member of the above equality is always included in the left one. Indeed let $x^{*} \in\left(\lim \sup _{x \in D \rightarrow{ }_{f} a} \underline{\partial} f(x)\right)$ with $x^{*}=w^{*} \lim x_{i}^{*}$, $x_{i}^{*} \in \underline{\partial} f\left(x_{i}\right)$ and $x_{i} \rightarrow{ }_{f} a$. 
Then we have $\left\langle x^{*}, h\right\rangle=\lim \sup _{i}\left\langle x_{i}^{*}, h\right\rangle \leq \lim \sup _{i} f^{0}\left(x_{i} ; h\right) \leq f^{0}(a ; h)$ for any $h \in E$. Therefore $\left\langle x^{*}, h\right\rangle \leq \liminf _{h^{\prime} \rightarrow h} f^{0}\left(a ; h^{\prime}\right)=f^{\dagger}(a ; h) \quad \forall h \in E$ (see Proposition 1.1), and hence $x^{*} \in \partial f(a)$. So for any

$$
z^{*} \in \operatorname{clco}\left(\limsup _{x \in D \rightarrow f^{a}} \underline{\partial} f(x)\right)
$$

and any $y^{*} \in N\left(\operatorname{dom} f^{\dagger}(a ;) ; 0.\right)$, we have $\left\langle z^{*}+y^{*}, h\right\rangle \leq f^{\dagger}(a ; h) \quad \forall h \in$ $\operatorname{dom} f^{\uparrow}(a ;$.$) which ensures that \left\langle z^{*}+y^{*}, h\right\rangle \leq f^{\dagger}(a ; h) \quad \forall h \in E$, and hence $z^{*}+y^{*} \in \partial f(a)$.

Let us now prove the condition is sufficient. We may suppose $\partial f(a) \neq \varnothing$. If we put $U=\{0\} U$ int $\operatorname{dom} f^{\dagger}(a ;$.$) , then by Lemma 2.1$

$$
f^{\dagger}(a ; h) \leq r(h)+\psi_{U}(h) \quad \forall h \in E\left(\psi_{U}\right. \text { denotes the indicator function). }
$$

Moreover by (4) and Lemma 2.1 we have $|r(h)|<\infty \quad \forall h \in \operatorname{int} \operatorname{dom} f^{\dagger}(a ;$.). As $\psi_{U}$ is continuous on int $\operatorname{dom} f^{\uparrow}(a ;$.$) we have \partial f^{\uparrow}(a ;).(0) \subset \partial r(0)+\partial \psi_{U}(0)$, which ensures that

$$
\partial f(a) \subset \operatorname{clco}\left(\limsup _{x \in D \rightarrow f^{a}} \underline{\partial} f(x)\right)+N\left(\operatorname{dom} f^{\dagger}(a ; .) ; 0\right) .
$$

The other implication is obvious.

\section{Clarke Subdifferentials IN Terms of $D$-Limiting gradients}

In this section we shall need the concept of strictly directionally Lipschitz functions.

3.1. Definition. We shall say that a function $f: E \rightarrow \mathbb{R} \cup\{+\infty\}$ is strictly directionally Lipschitz at a point $a \in \operatorname{dom} f$ if $f$ is directionally Lipschitz at $a$ and if for any vector $h$ with respect to which $f$ is directionally Lipschitz there exist $\alpha>0, k \in \mathbb{R}$, and $V \in \mathscr{N}(0)$ such that

(5)

$t^{-1}\left[f\left(x+t h^{\prime}\right)-f(x)\right] \leq k \quad$ for all $x \in V_{f}(a, \alpha), h^{\prime} \in h+V$, and $\left.t \in\right] 0, \alpha[$, and such that $f$ is locally Lipschitz over the set $\left.\left.V_{f}(a, \alpha)+\right] 0, \alpha\right](h+V)$, where $V_{f}(a, \alpha):=\{x \in E: x \in a+V,|f(x)-f(a)|<\alpha\}$.

Remark. If $f$ is a convex function which is continuous at some point, then $f$ is strictly directionally Lipschitz at any point $a \in \operatorname{dom} f$. This is the case if $f$ is l.s.c., $E$ is a Banach space, and int $\operatorname{dom} f \neq \varnothing$.

3.2. Lemma. Let $f: E \rightarrow \mathbb{R} \cup\{+\infty\}$ be l.s.c. on $E$ and strictly directionally Lipschitz at $a \in \operatorname{dom} f$. If $h \in \operatorname{int} \operatorname{dom} f^{\dagger}(a ;$.$) and if \alpha, k$, and $V$ are given by Definition 3.1, then for any $x \in V_{f}(a, \alpha)$ and any $h^{\prime} \in h+V$ the function $g:[0, \alpha] \rightarrow \mathbb{R}$ defined by $g(t)=f\left(x+t h^{\prime}\right)$ is continuous.

Proof. As $f$ is locally Lipschitz on $\left.\left.V_{f}(a, \alpha)+\right] 0, \alpha\right](h+V)$ we only need to prove that $g$ is continuous at 0 . By (5) we have $g(t) \leq g(0)+t k$ for all 
$t \in] 0, \alpha]$ which ensures by l.s.c. of $f$ that

$$
g(0) \leq \liminf _{t \downarrow 0} g(t) \leq \limsup _{t \downarrow 0} g(t) \leq g(0) .
$$

Let us consider now the notion of pseudoregular functions, which seems more suitable than that of regular functions whenever one deals with the $D$ representation of the Clarke subdifferential.

3.3. Definition. Let $f: E \rightarrow \mathbb{R} \cup\{+\infty\}$ be 1.s.c. on $E$ and directionally Lipschitz at $a$. We shall say $f$ is pseudoregular at $a$ if for each $h \in \operatorname{int} \operatorname{dom} f^{\dagger}(a ;$. there exist $\alpha>0$ and $V \in \mathscr{N}(0)$ such that for all $x \in V_{f}(a, \alpha), h^{\prime} \in h+V$, and $t \in] 0, \alpha]$ the restriction on $[0, \alpha]$ of the function $s \rightarrow f\left(x+s h^{\prime}\right)$ is continuous, and

$$
d^{+} f\left(x+t h^{\prime} ;-h^{\prime}\right)=f^{0}\left(x+t h^{\prime} ;-h^{\prime}\right) .
$$

Remarks. (1) if $f$ is l.s.c., convex, and continuous at some point, then it is not difficult to see that $f$ is pseudoregular at any $a \in \operatorname{dom} f$.

(2) If $f$ is locally Lipschitz around $a$, then $f$ is pseudoregular at $a$ iff for every $x$ in some neighborhood of $a$ one has $d^{+} f(x ;)=.f^{0}(x ;$.$) .$

(3) Obviously if $f$ is strictly directionally Lipschitz at $a$, then it is pseudoregular at $a$ whenever it is regular at $a$ (i.e. $\underline{d} f(x ;)=.f^{\dagger}(x ;$.$) for all$ $x$ in some neighborhood of $a$ ). However, the reverse does not hold even for Lipschitz functions. Indeed for $f(x)=x \sin \left[(\ln |x|)^{1 / 3}\right]$ we have $f^{0}(0 ; h)=$ $|h|=d^{+} f(0 ; h)$ but $d^{-} f(0 ; h)=-|h|$.

We can now state the main result of the paper.

3.4. Proposition. Let $f: E \rightarrow \mathbb{R} \cup\{+\infty\}$ be l.s.c. and let $D$ be a graphically dense subset of $\operatorname{dom} f$ over which $f$ is Hadamard differentiable. If $f$ is strictly directionally Lipschitz and pseudoregular at $a \in \operatorname{dom} f$, then

$$
\partial f(a)=\operatorname{cl} \operatorname{co}\left\{\lim \nabla f\left(x_{i}\right): x_{i} \in D \rightarrow{ }_{f} a\right\}+N\left(\operatorname{dom} f^{\uparrow}(a ; .) ; 0\right) .
$$

Proof. By Proposition 2.2 it is sufficient to prove that

$$
f^{\dagger}(a ; h) \leq \limsup _{x \in D \rightarrow f_{f} a}\langle\nabla f(x), h\rangle .
$$

Let $h \in \operatorname{int} \operatorname{dom} f^{\dagger}(a ;$.$) and let \alpha$ and $V$ satisfy relations (5) and (6). Choose $\varepsilon>0$. For $x \in V_{f}(a), h^{\prime} \in h+V$, and $\left.\left.t \in\right] 0, \alpha\right]$ we have, by Lemma 3.2 and Dini's mean value theorem (see [8]),

$$
\begin{aligned}
t^{-1}\left[f\left(x+t h^{\prime}\right)-f(x)\right] & =t^{-1}\left[(-f)\left(x+t h^{\prime}-t h^{\prime}\right)-(-f)\left(x+t h^{\prime}\right)\right] \\
& \left.\leq d^{-}(-f)\left(x+s h^{\prime} ;-h^{\prime}\right), \quad \text { with } s \in\right] 0, t[ \\
& =-d^{+} f\left(x+s h^{\prime} ;-h^{\prime}\right),
\end{aligned}
$$

and since $f$ is pseudoregular near $a$ we have

$$
d^{+} f\left(x+s h^{\prime} ;-h^{\prime}\right)=\limsup _{x^{\prime} \rightarrow f^{x+s h^{\prime}}} d^{+} f\left(x^{\prime} ;-h^{\prime}\right) \text {. }
$$


Therefore for such $x, h^{\prime}$, and $t$

$$
\begin{aligned}
t^{-1}\left[f\left(x+t h^{\prime}\right)-f(x)\right] & \leq-\limsup _{x^{\prime} \in D \rightarrow f^{x+s h^{\prime}}}\left\langle\nabla f\left(x^{\prime}\right),-h^{\prime}\right\rangle \\
& =\liminf _{x^{\prime} \in D \rightarrow f^{x+s h^{\prime}}}\left\langle\nabla f\left(x^{\prime}\right), h^{\prime}\right\rangle \\
& \leq \varepsilon+\limsup _{x^{\prime} \in D \rightarrow f_{f} x}\left\langle\nabla f\left(x^{\prime}\right), h^{\prime}\right\rangle,
\end{aligned}
$$

which completes the proof.

Consider now the case of convex functions.

3.5. Corollary. Let $f: E \rightarrow \mathbb{R} \cup\{+\infty\}$ be l.s.c., convex, and continuous at some point, and let $D$ be a dense subset of int $\operatorname{dom} f$ over which $f$ is Gateaux differentiable. Then for any $a \in \operatorname{dom} f$ we have

$$
\partial f(a)=\operatorname{clco}\left\{\lim _{i} \nabla f\left(x_{i}\right): x_{i} \in D \rightarrow a\right\}+N(\operatorname{dom} f ; a) .
$$

Proof. The function $f$ is strictly directionally Lipschitz at any $b \in \operatorname{dom} f$ (see the remark following Definition 3.1). Thus for a nonzero, $h \in \operatorname{int} \operatorname{dom} f^{\dagger}(b ;$.$) ;$ and some circled open neighborhood $V$ of zero relation (5) and the l.s.c. of $f$ easily imply that the restriction of $f$ on $H:=(b+[0 ; \alpha](h+V))$ is continuous at $b$. As $D \cap(H \backslash\{b\})$ is dense in $H$, there are $x_{i} \in D \rightarrow{ }_{f} b$. Therefore $D$ is graphically dense in $\operatorname{dom} f$.

Moreover, as int dom $\left.f^{\dagger}(a ;)=.\right] 0,+\infty[(\operatorname{int} \operatorname{dom} f-a)$ (see [11]) we have $x^{*} \in N\left(\operatorname{dom} f^{\dagger}(a ;) ; 0.\right)$ iff $\left\langle x^{*}, x-a\right\rangle \leq 0 \quad \forall x \in$ int dom $f$ and hence iff $x^{*} \in N(\operatorname{dom} f ; a)$. Therefore by Proposition 3.4 we obtain

$$
\begin{gathered}
\operatorname{clco}\left\{\lim _{i} \nabla f\left(x_{i}\right): x_{i} \in D \rightarrow a\right\}+N(\operatorname{dom} f ; a) \\
\subset \partial f(a)=\operatorname{clco}\left\{\lim _{i} \nabla f\left(x_{i}\right): x_{i} \in D \rightarrow{ }_{f} a\right\}+N(\operatorname{dom} f ; a) .
\end{gathered}
$$

So the proof is complete since the last set is obviously contained in the first one.

The following result has been proved by R. T. Rockafellar [10] when $E$ is finite dimensional. His method is different and less simple than ours, in the sense that on the one hand it uses the result of V. L. Klee about the representation of closed convex sets in terms of extremal points and extremal directions, and on the other hand it invokes some other finite dimensional arguments.

3.6. Corollary. Let $f: E \rightarrow \mathbb{R} \cup\{+\infty\}$ be an l.s.c. convex function defined on a weak Asplund Banach space $E$ with int $\operatorname{dom} f \neq \varnothing$. Then

$$
\partial f(a)=\operatorname{clco}\left\{\lim \nabla f\left(x_{i}\right): x_{i} \rightarrow a\right\}+N(\operatorname{dom} f ; a) \quad \forall a \in \operatorname{dom} f .
$$

Proof. As $E$ is a weak Asplund space (see [7]), the set $D:=\operatorname{dom} \nabla f$ is dense in int $\operatorname{dom} f$ and hence it suffices to apply Corollary 3.5 . 
Remark. As in [10] Corollary 3.6 can be used to study essentially smooth convex functions.

\section{REFERENCES}

1. J. Borwein and H. M. Strojwas, Proximal analysis and boundaries of closed set in space II: applications (to appear).

2. S. Chu-Chung, Remarques sur le gradient généralisé, J. Math. Pures Appl. 61 (1982), 301310.

3. F. H. Clarke, Generalized gradients and applications, Trans. Amer. Math. Soc. 205 (1975), 247-262.

4. __ Nonsmooth analysis and optimization, Wiley-Interscience, New York, 1983.

5. R. Correa and A. Jofre, Tangentially continuous directional derivatives in nonsmooth analysis, J. Optim. Theory Appl. (to appear).

6. R. Correa and L. Thibault, Subdifferential analysis of bivariate separately regular functions, J. Math. Anal. Appl. (to appear).

7. J. R. Giles, Convex analysis with application in the differentiation of convex functions, Pitman, 1982.

8. J. B. Hiriart-Urruty, A note on the mean value theorem for convex functions, Boll. Un. Mat. Ital. B 17 (1980), 765-775.

9. R. Mifflin, Semismooth and semiconvex functions in constrained optimization, SIAM J. Control. Optim. 15 (1977), 959-972.

10. R. T. Rockafellar, Convex analysis, Princeton Univ. Press, Princeton, NJ, 1970.

11. _. Generalized directional derivatives and subgradients of nonconvex functions, Canad. J. Math. 32 (1980), 257-280.

12. _ Directionally Lipschitzian functions and subdifferential calculus, Proc. London Math. Soc. 39 (1979), 331-355.

13. __ Proximal subgradients, marginal values, and augmented Lagrangians in nonconvex optimization, Math. Oper. Res. 6 (1981), 424-436.

14. L. Thibault, On generalized differentials and subdifferentials of Lipschitz vector-valued functions, Nonlinear. Anal. 6 (1982), 1037-1053.

15. J. S. Treiman, Clarke's gradients and epsilon-subgradients in Banach spaces, Trans. Amer. Math. Soc. 294 (1986), 65-78.

D’epartement de Mathématiques, Faculté des Sciences de Pau, 64000 Pau, France 